CASE REPORT

\title{
Gradual enhancement pattern of tracheobronchial adenoid cystic carcinoma on multiphasic dynamic computed tomography : a case series
}

\author{
Shinsaku Matsumoto ${ }^{1,2}$, Shogo Fukuma ${ }^{3}$, Takayoshi Shinya ${ }^{4,5,6}$, Takahiro Kitayama ${ }^{3}$, Soichiro Kajita ${ }^{3}$, \\ Yoshihisa Masaoka ${ }^{3}$, Shinji Hashimura ${ }^{7}$, Claus Peter Heusse ${ }^{5,6,8}$, Hans-Ulrich Kauczor ${ }^{5,8}$, and Susumu Kanazawa ${ }^{3}$ \\ ${ }^{1}$ Department of Diagnostic Radiology, Saiseikai Yokohamashi Tobu Hospital, 3-6-1 Shimosueyoshi, Tsurumi-ku, Yokohama City, Kanagawa, \\ 230-0012, Japan, ${ }^{2}$ Department of Diagnostic Radiology, Keio University School of Medicine, 35 Shinanomachi, Shinjuku-ku, Tokyo, 160-8582, \\ Japan, ${ }^{3}$ Department of Radiology, Okayama University Hospital, 2-5-1, Shikata-cho, Kita-ku, Okayama City, Okayama, 700-8558, Japan, ${ }^{4}$ De- \\ partment of Diagnostic and Therapeutic Radiology, Kawasaki Medical School General Medical Center, 2-6-2 Nakasange, Kita-ku, Okayama \\ City, Okayama, 700-8505, Japan, ${ }^{5}$ Department of Diagnostic and Interventional Radiology, University of Heidelberg, Germany, Im Neuen- \\ heimer Feld 110, Heidelberg, 69120, Germany, ${ }^{6}$ Department of Diagnostic and Interventional Radiology with Nuclear Medicine, Thoraxklinik \\ at University of Heidelberg, Röntgenstraße 1, Heidelberg, 69126, Germany, ${ }^{7}$ Department of Radiology, Okayama Red Cross Hospital, 2-1-1 \\ Aoe, Kita-ku, Okayama City, Okayama, 700-8607, Japan, ${ }^{8}$ Translational Lung Research Center Heidelberg (TLRC), Member of the German \\ Center for Lung Research (DZL), Im Neuenheimer Feld 110, Heidelberg, 69120, Germany
}

\begin{abstract}
Tracheobronchial adenoid cystic carcinoma is a rare malignancy. To the best of our knowledge, its enhancement pattern on multiphasic contrast-enhanced multidetector computed tomography has not been reported. Herein, we report the computed tomography findings of three consecutive cases of tracheobronchial adenoid cystic carcinoma. All lesions presented a gradual enhancement pattern on multiphasic contrast-enhanced computed tomography. We speculate that the gradual enhancement pattern on multiphasic contrast-enhanced computed tomography could potentially serve as a characteristic imaging feature and may therefore be a clue to diagnose tracheobronchial adenoid cystic carcinoma. J. Med. Invest. 67 : 378-381, August, 2020
\end{abstract}

Keywords : tracheobronchial adenoid cystic carcinoma, enhancement pattern, multiphasic contrast-enhanced CT

\section{INTRODUCTION}

Primary tracheal tumors are very uncommon. Adenoid cystic carcinoma (ACC) is the second most common primary malignancy of the trachea after squamous cell carcinoma (1). Generally, computed tomography (CT) is a useful and non-invasive modality for the detection and evaluation of tracheobronchial tumors. Although the imaging features of tracheobronchial ACC on contrast-enhanced CT have been previously reported (2-4), to the best of our knowledge, its enhancement patterns on multiphasic dynamic CT have not been described. Herein, we report the enhancement patterns on multiphasic dynamic CT of three consecutive cases of tracheobronchial ACC.

\section{CASE PRESENTATION}

Case 1

An 80-year-old man without a smoking history was referred to our hospital due to progressive dyspnea. The patient had been diagnosed with tracheal ACC and had undergone interventional tumor debulking (resection of $50 \%$ of the total tumor volume) and percutaneous radiotherapy (50 Gy in 25 fractions) 3 years previously at another hospital. We performed contrast-enhanced chest CT with an intravenous injection of $100 \mathrm{ml}$ of non-ionic iodinated contrast material containing $300 \mathrm{mg} \mathrm{I} / \mathrm{ml}$ at a rate of 2.0

Received for publication May 31, 2020 ; accepted June 25, 2020.

Address correspondence and reprint requests to Takayoshi Shinya, Department of Diagnostic and Therapeutic Radiology, Kawasaki Medical School General Medical Center, 2-6-2 Nakasange, Kita-ku, Okayama City, Okayama, 700-8505, Japan. $\mathrm{ml} / \mathrm{s}$. The scanning was performed as follows : (1) unenhanced images prior to contrast administration ; (2) $30 \mathrm{~s}$ after the start of contrast administration (early phase) ; and (3) $90 \mathrm{~s}$ after the start of contrast administration (late phase). CT revealed sub-circumferential wall thickening, mainly of the left wall, and an intraluminal lesion originating in the left wall of the intrathoracic lower trachea, with narrowing of the tracheal lumen. The lesion demonstrated a relatively homogeneous density and showed gradual enhancement in the dynamic study (Fig. 1 a-c). For measuring the attenuation values, the regions of interest (ROIs) were placed on the most avidly enhanced parts of the lesion that were devoid of vessels and calcifications. The mean attenuation value within each ROI was 65 Hounsfield Unit (HU) on the unenhanced, $76 \mathrm{HU}$ on the early-phase, and $117 \mathrm{HU}$ on the late-phase images. The attenuation difference between the late and unenhanced phases ( $\left.\mathrm{A}_{\mathrm{L}-\mathrm{Au}}\right)$ was $52 \mathrm{HU}$. Recurrence of ACC was therefore suspected. Bronchoscopy revealed a raised lesion in the left wall of the trachea and bronchoscopic biopsy was performed. The histopathologic analysis revealed that the tumor was composed of small cuboidal cells with a high nucleus/cytoplasm ratio and a cribriform structure. Recurrence of ACC was diagnosed. Tumor ablation with an argon plasma coagulator was performed and the patient's symptoms improved.

Case 2

A 70-year-old woman without a smoking history was referred to our hospital due to cough and wheezing persisting for several days. Although treatment for bronchial asthma attack was administered, no improvement was observed. The patient underwent multiphasic contrast-enhanced CT, which revealed a 7.0-cm long, smooth, semi-circular wall thickening on the right wall of the trachea extending to the bifurcation, with narrowing of the tracheal lumen. The lesion demonstrated a relatively homo- 
geneous density and showed gradual enhancement on dynamic CT (Fig. 2 a-c). The mean attenuation value within each ROI was $44 \mathrm{HU}$ on the unenhanced, $93 \mathrm{HU}$ on the early-phase, and 147 HU on late-phase images. The AL-Au was 103 HU. Bronchoscopy revealed a smooth-surfaced tumor with redness on the right tracheal wall extending to the bifurcation. Bronchoscopic biopsy was performed and the histopathologic analysis revealed proliferating small atypical cells in the subepithelial layer showing a cribriform pattern. On immunohistochemistry, the proliferating cells were positive for CK7, SMA (partly), and S100 (partly) and negative for CK20, vimentin, and p63. ACC was thus diagnosed. Surgical resection was considered to be difficult due to the wide extent of the tumor. Therefore, percutaneous radiotherapy (60 Gy in 30 fractions) and chemotherapy (TS-1) were performed. CT 2 months after the end of radiotherapy showed tumor shrinkage and reduced luminal narrowing. No tumor recurrence has been observed 2 years after the diagnosis.

Case 3

A 60 -year-old man with a smoking history of 400 pack-years was referred to our hospital due to dyspnea. Capillary oxygen saturation was $91 \%$ (room air), while the routine laboratory analysis was normal. Chest X-ray showed total obstruction atelectasis of the left lung. Subsequent multiphasic contrast-enhanced CT revealed a $3.8-\mathrm{cm}$ long smooth mass occupying the left main bronchus with consecutive obstruction atelectasis of the left lung. The mass displayed a relatively homogenous contrast enhancement, with gradual enhancement in the dynamic study (Fig. 3 a-c). The mean attenuation value within each ROI was $81 \mathrm{HU}$ on the unenhanced, $120 \mathrm{HU}$ on the early-phase, and $155 \mathrm{HU}$ on the late-phase images. The $\mathrm{AL}_{\mathrm{L}} \mathrm{Au}_{\mathrm{u}}$ was $74 \mathrm{HU}$. Bronchoscopy revealed a smooth mass with left main bronchus obliteration. Biopsy was not performed because the tumor was reddish, and vasodilatation was observed on its surface; thus, we considered there was a high risk of bleeding. As malignancy was suspected, left pneumonectomy was performed. The histopathological analysis revealed a submucosal tumor spreading throughout the entire bronchial circumference and the tumor cells showed a cribriform pattern. The patient was diagnosed with ACC. Because R1-resection was determined at the proximal bronchial stump, radiotherapy (66 Gy in 33 fractions) was applied additionally. Tumor recurrence has not been observed 7 years after the diagnosis.

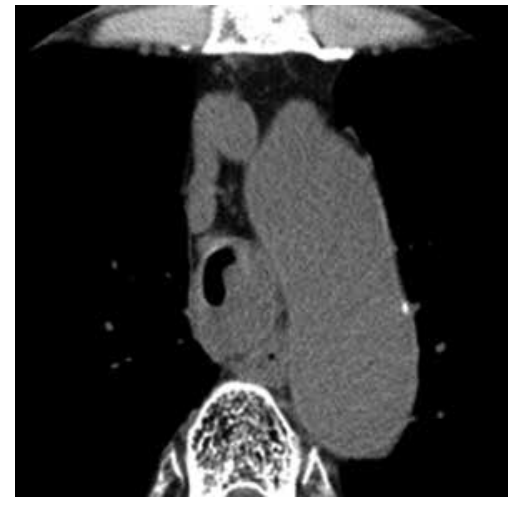

$1 \mathrm{a}$

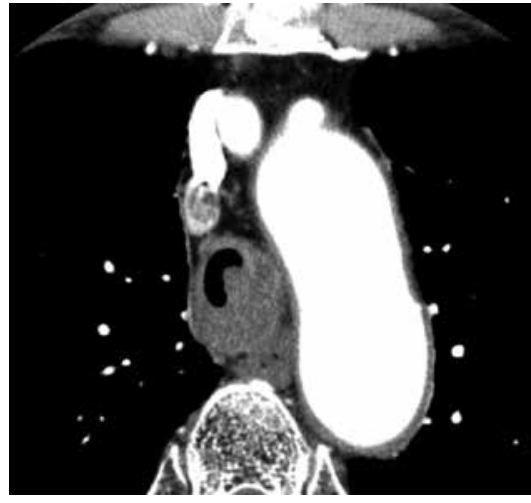

$1 \mathrm{~b}$

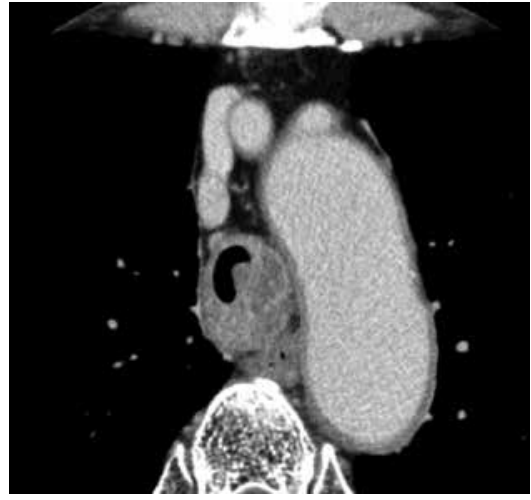

$1 \mathrm{c}$

Fig 1. Multiphasic contrast-enhanced computed tomography (CT) images of an 80-year-old man with adenoid cystic carcinoma. $(a-c)$ The tumor appears as a sub-circumferential wall thickening mainly of the left tracheal wall, showing gradual enhancement on multiphasic contrast-enhanced CT images.

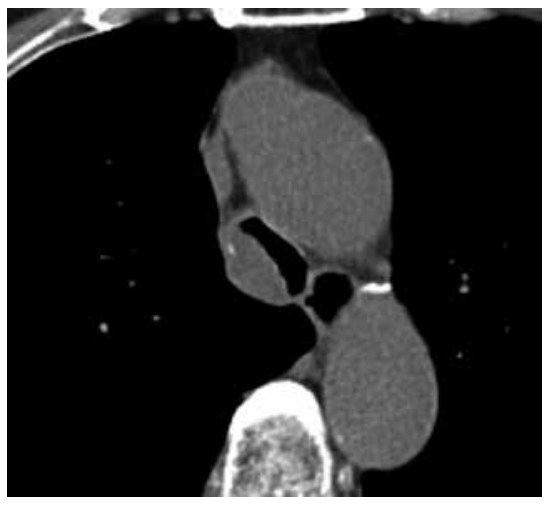

$2 a$

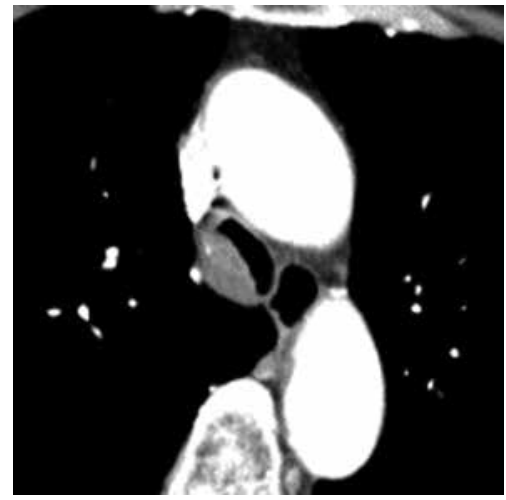

$2 b$

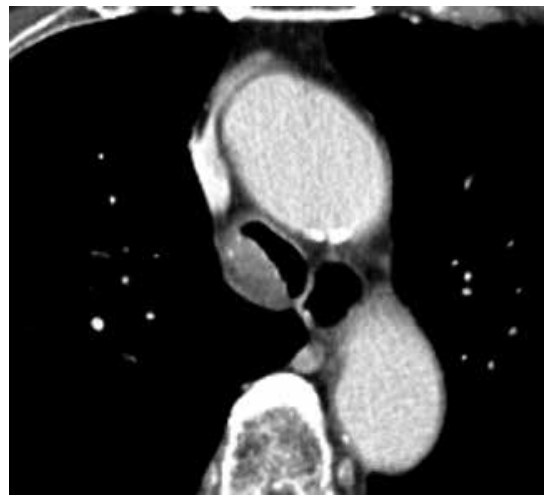

2c

Fig 2. Multiphasic contrast-enhanced computed tomography (CT) images of a 70-year-old woman with adenoid cystic carcinoma. $(\mathrm{a}-\mathrm{c})$ The tumor appears as a semi-circular tracheal wall thickening, showing gradual enhancement on multiphasic contrast-enhanced CT images. 


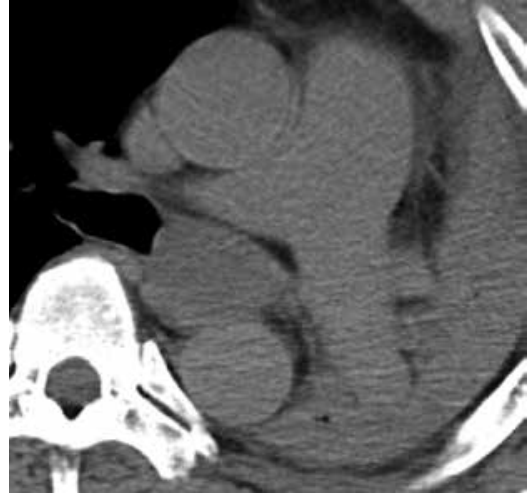

$3 a$

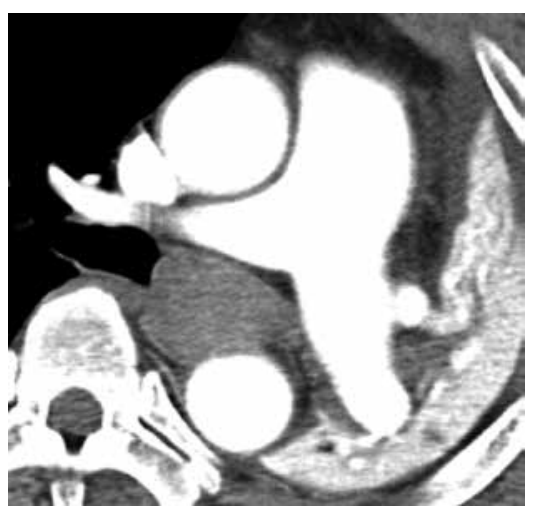

$3 b$

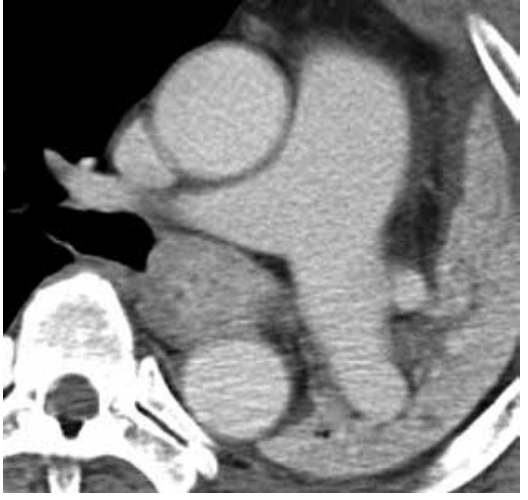

$3 c$

Fig 3. Multiphasic contrast-enhanced computed tomography (CT) images of a 60-year-old man with adenoid cystic carcinoma.

$(\mathrm{a}-\mathrm{c})$ The tumor appears as a 38-mm-long smooth mass occupying the left main bronchus and showing gradual enhancement on multiphasic contrast-enhanced CT images.

\section{DISCUSSION}

Tracheobronchial ACC is a rare low-grade tumor originating from the submucosal minor salivary glands of the tracheobronchial tree. It mainly arises in the lower trachea or main bronchi, causing obstruction atelectasis and dyspnea and is rarely found in the peripheral bronchi or lung parenchyma. Surgical resection is the first choice for treatment. Due to its submucosal nature, local tumor cell spread is often more extensive than expected prior to surgery $(5,6)$.

On chest CT, most ACCs manifest as a focal endoluminal mass or wall thickening. Due to the submucosal extension, the tumor may involve more than half of the airway circumference. Smooth surface and longitudinal extension along the airways are also typical features $(2,3,7,8)$.

Although it was previously reported that tracheobronchial ACC generally showed mild or moderate enhancement after the intravenous administration of contrast material $(2,3)$, the enhancement patterns on multiphasic dynamic CT have not been described. Furthermore, the most common morphological findings were thickened walls involving more than half of the airway circumference and longitudinal extension (2). However, in the early stage, the lesion can be seen as a polypoid intraluminal nodule $(2,9)$, and differentiation from other tracheobronchial tumors, including hemangioma, squamous cell carcinoma, mucoepidermoid carcinoma, and carcinoid tumors, is generally difficult based only on morphologic CT findings. In Case 3, morphological differentiation from other tracheobronchial tumors on CT was difficult even in retrospective evaluations because the tumor appeared as a nonspecific intraluminal polypoid mass. However, it showed a relatively homogeneous density and gradual progressive enhancement on dynamic CT, as did tumors in Cases 1 and 2.

The diagnostic utility of the enhancement pattern on multiphasic contrast-enhanced CT has been previously reported for malignant hepatic tumors, renal cell carcinomas, small bowel neoplasms, and lung cancer (10-13). In a recent article (13), Fukuma et al. demonstrated the enhancement pattern of 48 squamous cell carcinomas, and the average $\mathrm{HU}$ was $33.94 \mathrm{HU}$ in the unenhanced, $75.40 \mathrm{HU}$ in the early, and $92.01 \mathrm{HU}$ in the late phase. In contrast, in the present cases, the average HU on dynamic CT scans for three adenoid cystic carcinomas was 63.33 HU in the unenhanced, $96.33 \mathrm{HU}$ in the early, and $139.67 \mathrm{HU}$ in the late phase (Fig. 4). Moreover, all ACCs presented a gradual enhancement pattern with a severe peak enhancement of more than $110 \mathrm{HU}$ in the late phase. We suspect that the gradual and progressive enhancement pattern during multiphasic contrast-enhanced CT would be a characteristic imaging feature and may be a clue to diagnose tracheobronchial ACC based on imaging examination in cases when biopsy of the tracheobronchial tumor is not possible, as in our Case 3 . In addition, we speculated that tracheobronchial ACC could show high enhancement in the late phase $90 \mathrm{~s}$ after initiating contrast administration, and the AL-Au could reach over $100 \mathrm{HU}$ in some cases, as in our Case 2.

In conclusion, the gradual and progressive enhancement patterns on multiphasic contrast-enhanced CT could potentially serve as an important indicator of tracheobronchial ACC in addition to the morphological features. However, a study with a larger patient population that includes other type of tracheobronchial tumors is necessary to determine how often this gradual enhancement pattern occurs in ACC and how it can contribute to the differential diagnosis of tracheobronchial tumors.

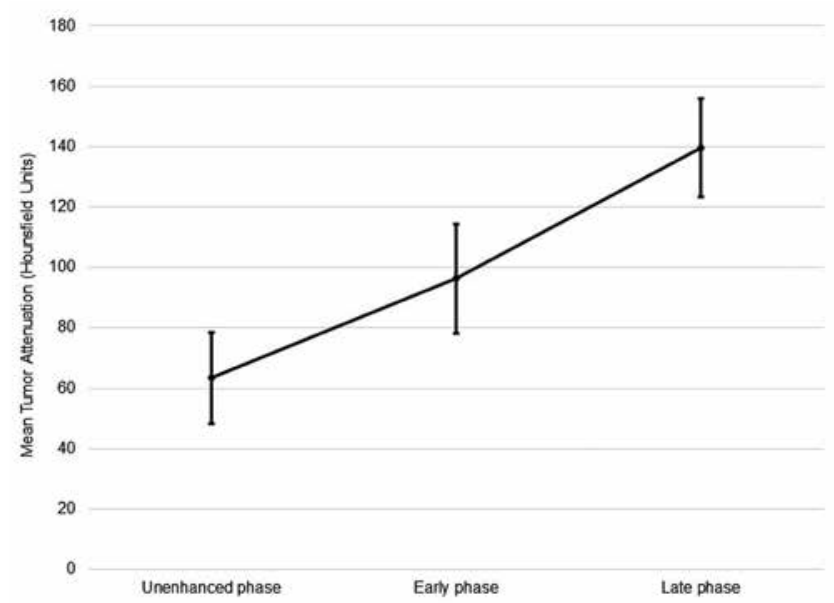

Fig 4. Mean attenuation value of the tumors in each phase.

The error bars show the standard deviation. The average Hounsfield unit (HU) was $63.33 \mathrm{HU}$ in the unenhanced, $96.33 \mathrm{HU}$ in the early, and $139.67 \mathrm{HU}$ in the late phase. 


\section{DECLARATION OF CONFLICTING INTERESTS}

The author(s) declared no potential conflicts of interest with respect to the research, authorship, and/or publication of this article.

\section{FUNDING}

The author(s) received no financial support for the research, authorship, and/or publication of this article.

\section{REFERENCES}

1. Urdaneta AI, Yu JB, Wilson LD : Population based cancer registry analysis of primary tracheal carcinoma. Am J Clin Oncol 34 : 32-37, 2011

2. Li X, Yi W, Zeng Q: CT features and differential diagnosis of primary pulmonary mucoepidermoid carcinoma and pulmonary adenoid cystic carcinoma. J Thorac Dis $10: 6501$ 6508, 2018

3. Han X, Zhang J, Fan J, Cao Y, Gu J, Shi H : Radiological and clinical features and outcomes of patients with primary pulmonary salivary gland-type tumors. Can Respir J 1475024, 2019

4. Elnayal A, Moran CA, Fox PS, Mawlawi O, Swisher SG, Marom EM : Primary salivary gland-type lung cancer: imaging and clinical predictors of outcome. Am J Roentgenol 201 : 57-63, 2013

5. Pandey D, Garg PK, Jakhetiya A, Pandey R, Bhoriwal S, Nath D, Kumar S : Surgical experience of primary salivary gland tumors of lung : a case series. Int J Surg 21 : 92-96, 2015

6. Calzada AP, Miller M, Lai CK, Elashoff DA, Abemayor E, St John MA : Adenoid cystic carcinoma of the airway : a 30-year review at one institution. Am J Otolaryngol $33: 226-231,2012$

7. Kwak SH, Lee KS, Chung MJ, Jeong YJ, Kim GY, Kwon OJ : Adenoid cystic carcinoma of the airways : helical CT and histopathologic correlation. AJR Am J Roentgenol $183: 277-281,2004$

8. Park CM, Goo JM, Lee HJ, Kim MA, Lee CH, Kang MJ : Tumors in the tracheobronchial tree: CT and FDG PET features. Radiographics 29: 55-71, 2009

9. Dean CW, Speckman JM, Russo JJ : AIRP best cases in radiologic-pathologic correlation : adenoid cystic carcinoma of the trachea. Radiographics $31: 1443-1447,2011$

10. Tsunematsu S, Chuma M, Kamiyama T, Miyamoto N, Yabusaki S, Hatanaka K, Mitsuhashi T, Kamachi H, Yokoo H, Kakisaka T, Tsuruga Y, Orimo T, Wakayama K, Ito J, Sato F, Terashita K, Nakai M, Tsukuda Y, Sho T, Suda G, Morikawa K, Natsuizaka M, Nakanishi M, Ogawa K, Taketomi A, Matsuno Y, Sakamoto N : Intratumoral artery on contrast-enhanced computed tomography imaging : differentiating intrahepatic cholangiocarcinoma from poorly differentiated hepatocellular carcinoma. Abdom Imaging $40: 1492-1499,2015$

11. Jinzaki M, Tanimoto A, Mukai M, Ikeda E, Kobayashi S, Yuasa Y, Narimatsu Y, Murai M : Double-phase helical CT of small renal parenchymal neoplasms : correlation with pathologic findings and tumor angiogenesis. J Comput Assist Tomogr 24 : 835-842, 2000

12. Shinya T, Inai R, Tanaka T, Akagi N, Sato S, Yoshino T, Kanazawa S : Small bowel neoplasms: enhancement patterns and differentiation using post-contrast multiphasic multidetector CT. Abdom Radiol (NY) 42 : 794-801, 2017

13. Fukuma S, Shinya T, Soh J, Fukuhara R, Ogawa N, Higaki F, Tanaka T, Ichihara E, Hiraki T, Toyooka S, Kanazawa $\mathrm{S}$ : Association between histological types and enhancement of dynamic CT for primary lung cancer. Acta Med Okayama 74 : 129-135, 2020 